\title{
BIOLOGICAL AND PROSTHETIC COMPLICATIONS OF CANTILEVERED AND NON-CANTILEVERED FIXED HYBRID METAL ACRYLIC PROSTHESES USED FOR VERTICAL AND INCLINED IMPLANTS IN ATROPHIED MANDIBLE. A RANDOMIZED CLINICAL TRIAL
}

\author{
Ahmed Samir Khairallah* and Mohammed Omran Hamed**
}

\begin{abstract}
Aim: This study aimed to evaluate biological and prosthetic complications of cantilevered and non-cantilevered fixed hybrid metal acrylic prostheses used for vertical and inclined implants in atrophied mandible.
\end{abstract}

Materials and Methods: Eight completely edentulous individuals will be classified into 2 groups randomly: 1) Group I (cantilevered prosthesis, $n=4$ ): patients received 4 vertical implants parallel to each other's in the interforaminal area and cantilevered metal acrylic prosthesis, 2) Group II (non-cantilevered prosthesis, $n=4)$ : patients received 4 implants (2 parallel anterior implants and 2 posterior implants inclined $30^{\circ}$ distally) and non-cantilevered metal acrylic prosthesis. Biological and prosthetic complications were measured after one year from prosthesis insertion.

Results: There was no significant difference in implant failure, implant survival, pain, swelling, abscess, fistula formation, peri-implantitis, peri-implant mucositis, bleeding on probing between groups. The non-cantilevered group showed significantly higher pocket depth than the cantilevered group. The cantilevered group showed significantly higher bone loss than the non-cantilevered group. There were no prosthesis fractures, cylinder fracture, abutment fracture, and prosthetic screw fracture occurred in both groups. There was no difference in teeth wear, teeth fracture, and gingival fracture between groups. The cantilevered group showed a significantly higher crown fracture, abutment screw, and prosthetic loosening than the non-cantilevered group.

Conclusion: Both cantilevered and non-cantilevered fixed hybrid metal acrylic prostheses can be used successfully for vertical and inclined implants respectively in atrophied mandible with favorable biologic and prosthetic outcomes after one year. However, with exception of increased pocket depth, the non-cantilevered group was advantageous regarding reduced bone loss, crown fracture, abutment, and prosthetic screw loosening, compared to the cantilevered group.

\footnotetext{
* Department of Removable Prosthodontics Mansoura Faculty of Dentistry

** OMFS, Faculty of Oral and Dental Medicine, Beni-Suef University, Beni-Suef, Egypt
} 


\section{INTRODUCTION}

Implant-supported prosthesis is considered a viable option for edentulous patients as it improves prosthesis stability, masticatory efficiency, and oral health related quality of life compared to conventional dentures ${ }^{1}$. Atrophic mandible poses a significant challenge to successful oral rehabilitation with dental implants ${ }^{2}$. Although ridge augmentation can help to restore ridge volume, grafting procedures can significantly increase patient morbidity, costs, and treatment time ${ }^{3,4}$. The full-arch screw-retained hybrid prosthesis was used with atrophic mandible with inadequate bone height in the posterior ridges above the mandibular canals to avoid important anatomical structures (inferior alveolar nerve). Mandibular implant-supported screw-retained hybrid fixed prostheses have been advocated originally by Brånemark for edentulous patients who had problems with conventional dentures ${ }^{5}$. Such prosthesis is fabricated on 4 or 5 implants inserted parallel to each other's in the interforaminal region of the mandible and consisted of cast metal frameworks, denture bases, and denture teeth and retained by screws threaded into the implant abutments. The anterior part of the prosthesis is fixed on implants while the posterior part is cantilevered from implants ${ }^{6}$. The hybrid prosthesis has fewer posterior teeth than a conventional complete denture ${ }^{7}$. Cantilever lengths of 1.5 times the A/P spread were decided empirically for such prostheses after considering clinical conditions that might biomechanically compromise the biologic and/or prosthetic outcomes of clinical cases ${ }^{8}$. In a more recent study, the authors noted that fabricating the frameworks for screw retained, hybrid prostheses with cantilever length/Antroposterior spread ratios less than 1.0 resulted in virtually complication-free prostheses ${ }^{9}$.

The "All on four" implant concept for rehabilitation of edentulous patient has gained popularity in the last 2 decades with high cumulative implant and prostheses survival rates $(96.7 \%$ and $98.2 \%$ ) that can be compared to the conventional two-stage protocols ${ }^{10-12}$. The concept includes installing 2 implants in the canine or lateral incisor positions parallel to each other's and vertically in the mandible. The posterior implants are inclined distally to make an angle of 30-45o from the vertical planes. In the mandible, the posterior implants are placed just anterior to the mental foramen. The concept has several merits such as: 1) reduction of the necessity to used bone grafting/ augmentation or nerve repositioning in the mandible or use sinus left procedure in the maxilla, thus it is suitable for medically compromised patients with reduced morbidity, 2) immediate loading of the implants, immediate restoration of mastication and esthetics by the provisional acrylic dentures, 3) cost effectiveness due to reduction of the number of used implants $^{12,13}$, 4) Placement of longer posterior implants due to tilting of the implants which increase surface area and bone to implant contact, 5) wide implant support which allow placement of short distal cantilevers, and 6) reduction of the duration of the treatment (time saving procedure) $)^{14,15}$

Implant success and survival is not only the key of success of a specific type of rehabilitation, but also biological and prosthetic complications play an important role ${ }^{16}$. Implant and prosthetic complications have been a constant struggle for restorative dentists, surgeons, and patients alike. Implant-related problems can be particularly challenging and frustrating, especially given that an implant is thought to be a "lifetime" solution expected to yield minimal difficulties ${ }^{17}$. Reviewing the literature, there is a lack of studies about the incidence of complications and their implications with metal acrylic fixed prostheses used for vertical and inclined implants. Therefore, the aim of this study was to evaluate potential prosthetic and biologic complications with cantilevered and noncantilevered fixed hybrid metal acrylic prostheses used for vertical and inclined implants in atrophied 
mandible. The null hypothesis is no significant difference in complications between the 2 groups would be expected.

\section{MATERIALS AND METHODS}

\section{Patient cohort and study design}

Eight completely edentulous individuals (4 males and 4 females) with mean age of $62.8 \pm 4.3$ years were included in this study from outpatient clinic of Prosthodontic Department. The participants had the following criteria: 1) atrophied mandibular ridges (class IV-VI) according to the classification proposed by $\left(\right.$ Cawood \& Howell $\left.{ }^{18}\right)$ with insufficient retention and patient complain from instability of mandibular dentures, 2) sufficient bone height and width in the interforaminal area to receive four implants with standard diameter (at least 11 $\mathrm{mm}$ long and $3.7 \mathrm{~mm}$ wide). The protocol of the study was reviewed and approved by the Faculty of dentistry Bani-suef university research Ethics committee (Approved number Approval number: \#FDBSUREC/09112020/KA. The exclusion criteria were: 1) serious problems of coagulation,2) diseases of the immune system, 3) uncontrolled diabetes, 4) metabolic diseases affecting bone, 5) irradiation of the head or neck region in the last 2 years, and 6) inadequate oral hygiene level. The patients instructed about the treatment protocol and objectives prior to obtain an informed consent. The participants were classified into 2 groups randomly: 1) Group I (cantilevered prosthesis): included 4 patients who received 4 vertical implants parallel to each other's in the interforaminal area and cantilevered hybrid metal acrylic prosthesis, 2) Group II (noncantilevered prosthesis): included 4 patients who received 4 implants in the interforaminal area (2 parallel anterior implants and 2 posterior implants inclined 30 degree distally according to the All on four concept) and non-cantilevered hybrid metal acrylic prosthesis. Allocation of the patients to treatment groups was made using a quasi-random method by random generated numbers generated in Excel sheet. The randomization was done in a manner that ensure equal gender distribution in both groups ( 2 males and 2 females in each group).

\section{Surgical and prosthetic procedures}

All patients received new maxillary and mandibular conventional dentures. The new mandibular denture was duplicated into acrylic resin radiographic template (with gutta perchae radiopaque markers fitted in the center of the artificial teeth). Patients underwent CT scans (CBCT, i- CAT Vision ${ }^{\circledR}$, Imaging Sciences International, Hatfield, PA, USA) to accurately assess the quantity of bone and location of mental foramen, mental loop and mandibular canal. All patients were sedated with diazepam prior to surgery. Antibiotics (amoxicillin $625 \mathrm{mg}+$ clavulanic acid $125 \mathrm{mg}$, Augmentin ${ }^{\circledR}$ $1 \mathrm{gm})$ were given 1 hour prior to surgery and daily for 6 days thereafter. Cortisone medication (Dexamethazone ${ }^{\circledR}$ ) was given. Anti-inflammatory medication (ibuprofen ${ }^{\circledR}, 600 \mathrm{mg}$ ) was administered for 4 days postoperatively. Analgesics (Ketolac $\AA$ $10 \mathrm{mg}$ ) were given on the day of surgery and postoperatively for the first 4 days. A mid crestal incision was made and a mucoperiosteal flap was raised to expose the crest and the mental foramina. When needed bone flattening was made to provide a flat shelf that provide at least $1 \mathrm{~mm}$ of bone buccal and lingual to each implant ${ }^{19}$.

Using a special metal guide (Malo edentulous guide $^{\mathrm{TM}}$, Nobel Biocare AB), patients in group I received 4 vertical implants (TioLogic, Dentaurum) parallel to each other's with the posterior implants located just anterior to the mental foramina. For group II, patients received 4 implants according to the "All-on-4 concept" (Malo, et al. 2003a) (fig 1).

Anterior implants were inserted at canine/ lateral incisor area parallel to each other and perpendicular to occlusal plane. Posterior implants were inserted in premolar area just anterior to mental foramina 


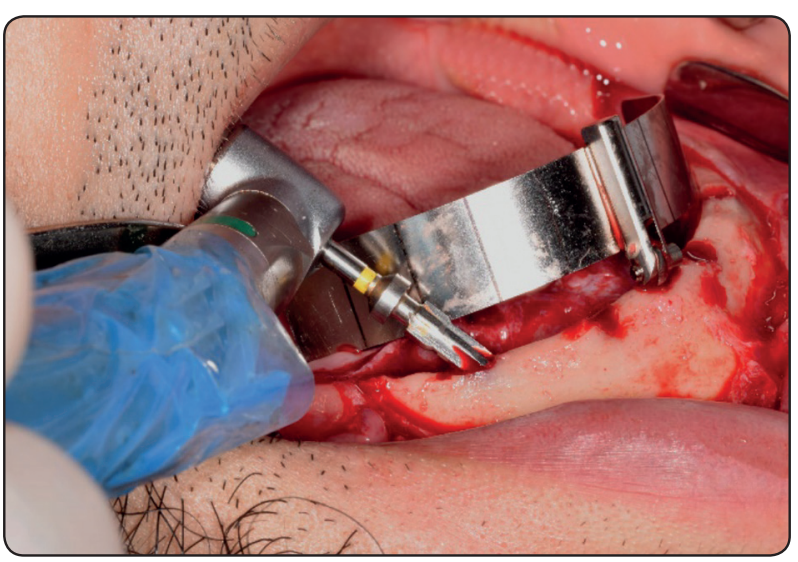

Fig. (1) Implant drilling using the metal guide

and tilted $30-35^{\circ}$ distally to emerge in the region of mesial cusp of the first molar tooth. This placement enhances good implant anchorage and support, eliminate the need of cantilever, and increases anteroposterior spread (Krekmanov, et al. 2000). Cover screws were threaded into the implant fixtures and the flap was closed with interrupted sutures. Post-operative panoramic radiographs were made for both groups (fig 2). The implants were left submerged for 3 months to allow osseointegration. The dentures were relieved and relined with resilient liners and the patients use them during the healing period. After 3 months, the implants in both groups were uncovered. Straight multiunit abutments (AngleFix abutments, TioLogic, Dentaurum) were used for vertical implants in both groups and 30-degree multiunit abutment were screwed into posterior implants in group II. All abutments were torqued at $25 \mathrm{Ncm}$.

Abutment level open tray impression procedure was made using a polyvinilsyloxane (Zhermack ${ }^{\circledR}$, Badia Polesine, Rovigo, Italy). To minimize movement of the transfer coping during impression making, the copings were splinted with ligature wire and Duralay autopolymerized resin pattern (Duralay, Reliance Dental MFG Co, Worth, IL, USA) on the casts. The splinting resin bars between the implants were sectioned and assembled intraorally to obtain passive fit before making the impressions. On the cast, the plastic caps were connected to multiunit abutments. The caps were connected to each other's using a resin bar. For group I, distal cantilevers were made (the length of each cantilever= antroposterior spread distance ${ }^{20}$, mean cantilver length was $14.2 \pm 2.1 \mathrm{~mm})$. For group II, no cantilevers were made. The plastic cap-resin bar assembly was cast in a nonprecious cobalt-chromium alloy (Heraenium Pw, Heraeus-Kulzer GmbH, Hanau, Germany). The cast superstructure was tried intraorally for passivity using the single screw Sheffield test (fig 3).

The cast bar was returned to the model and acrylic semi-anatomic artificial teeth were set at correct vertical dimension and centric relation. The lost alveolar bone and gingival tissue were
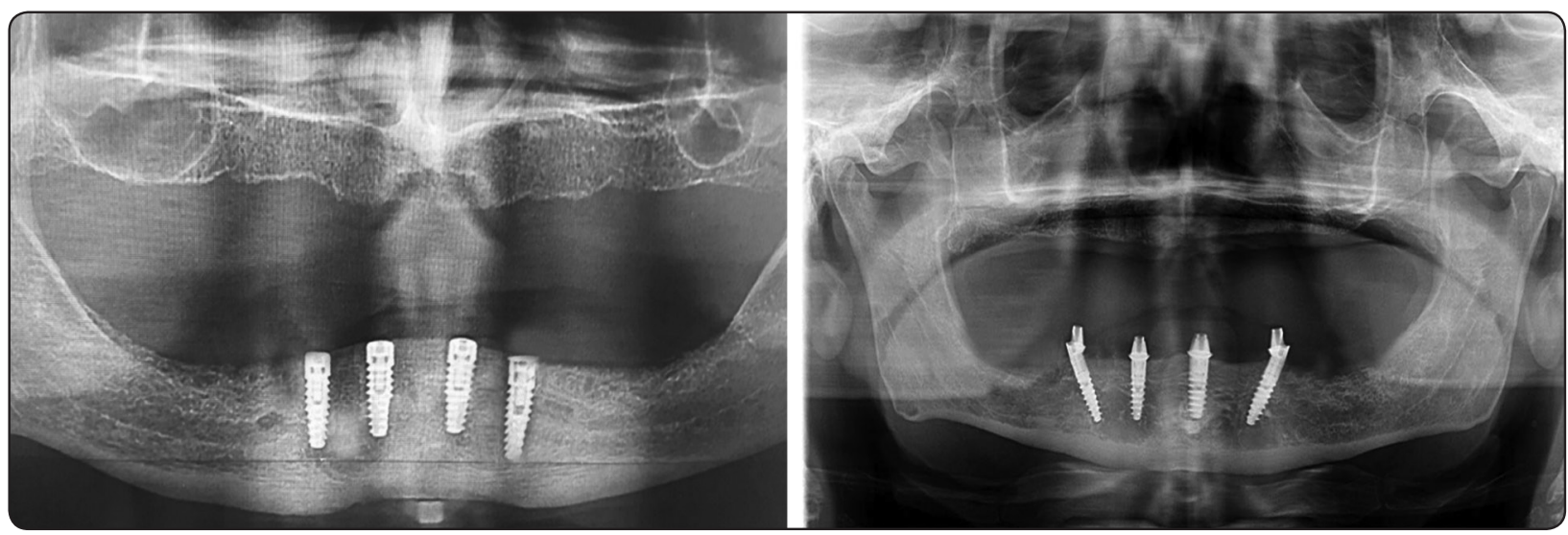

Fig. (2) Post-operative panoramic radiographs A) group I (vertical implants, cantilevered prosthesis), B) group II (inclined implants and non-cantilevered prosthesis) 
replaced by waxing up. The model was flasked, and the packing of acrylic rein was made in the usual manner. Finishing and polishing of the prosthesis after the restoration of access holes for prosthetic screws was made. The fixed partial denture has 12- unit artificial teeth in both groups ${ }^{7}$ with cantilevers in group I (Fig 4) and no cantilever in group II (Fig 5). The final prosthesis was delivered typically 3 months postsurgically. The screws access holes were sealed with composite resin ${ }^{21}$

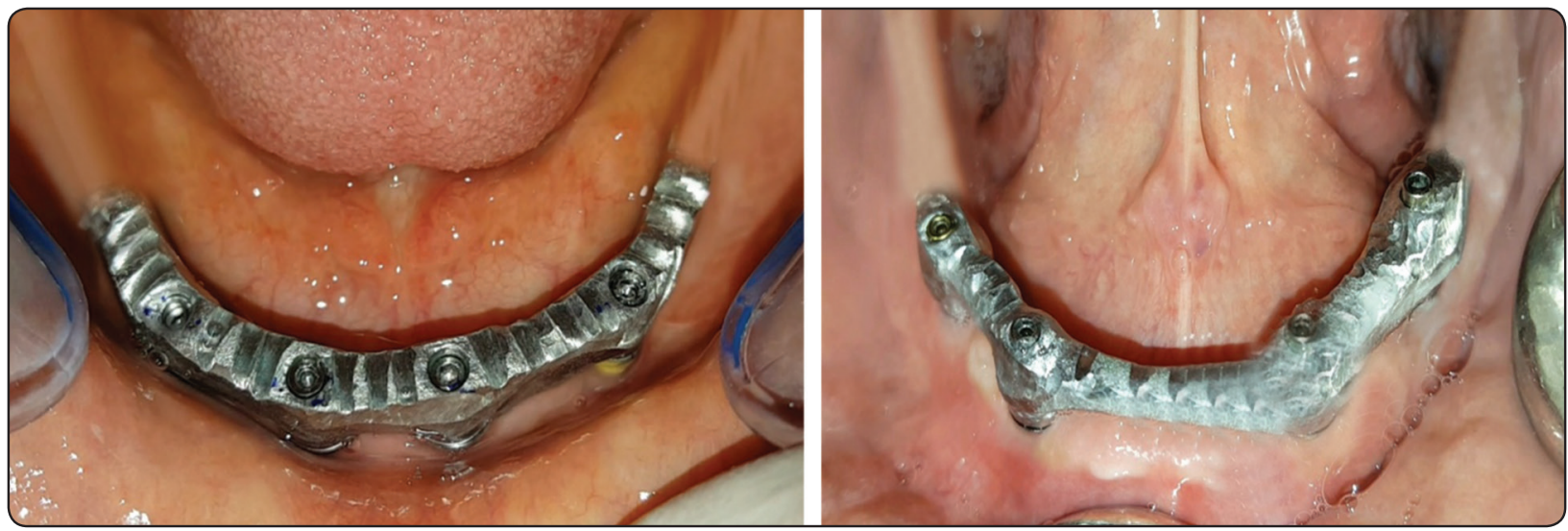

Fig. (3): Try in for passivity of the metal casting A) group I (vertical implants, cantilevered prosthesis), B) group II (inclined implants and non-cantilevered prosthesis)

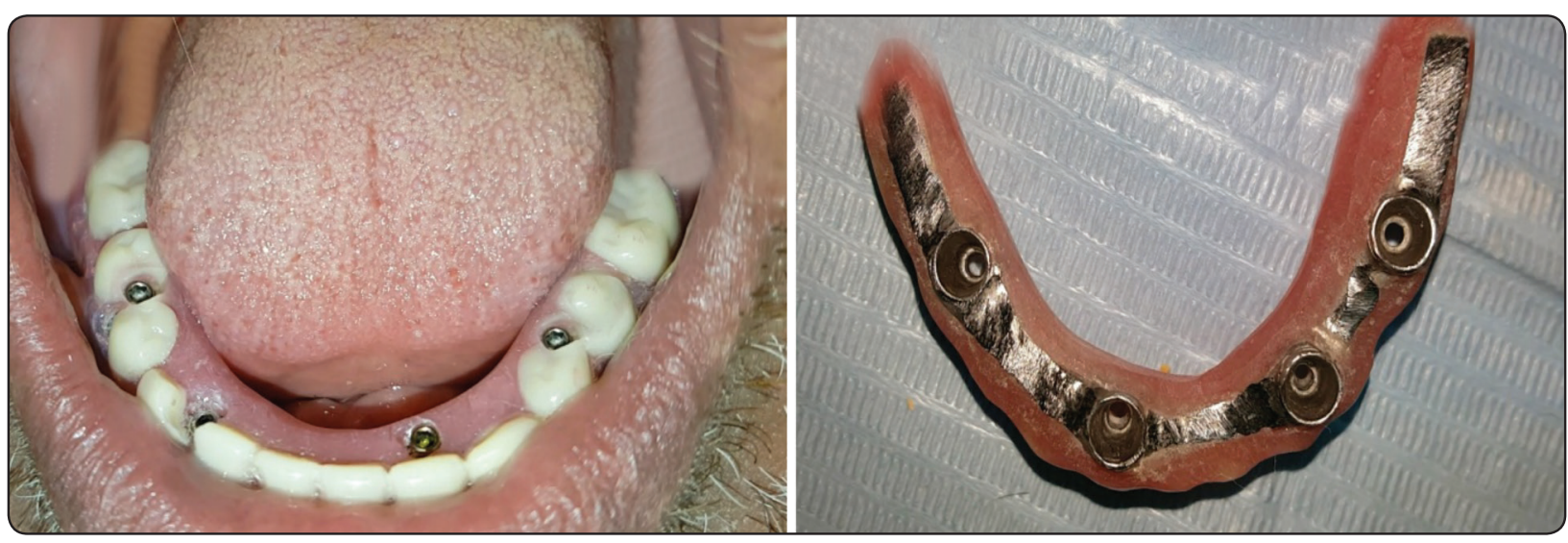

Fig. (4) Group I (vertical implants, cantilevered prosthesis), A) Intra-oral view, B) Fitting surface of the prosthesis

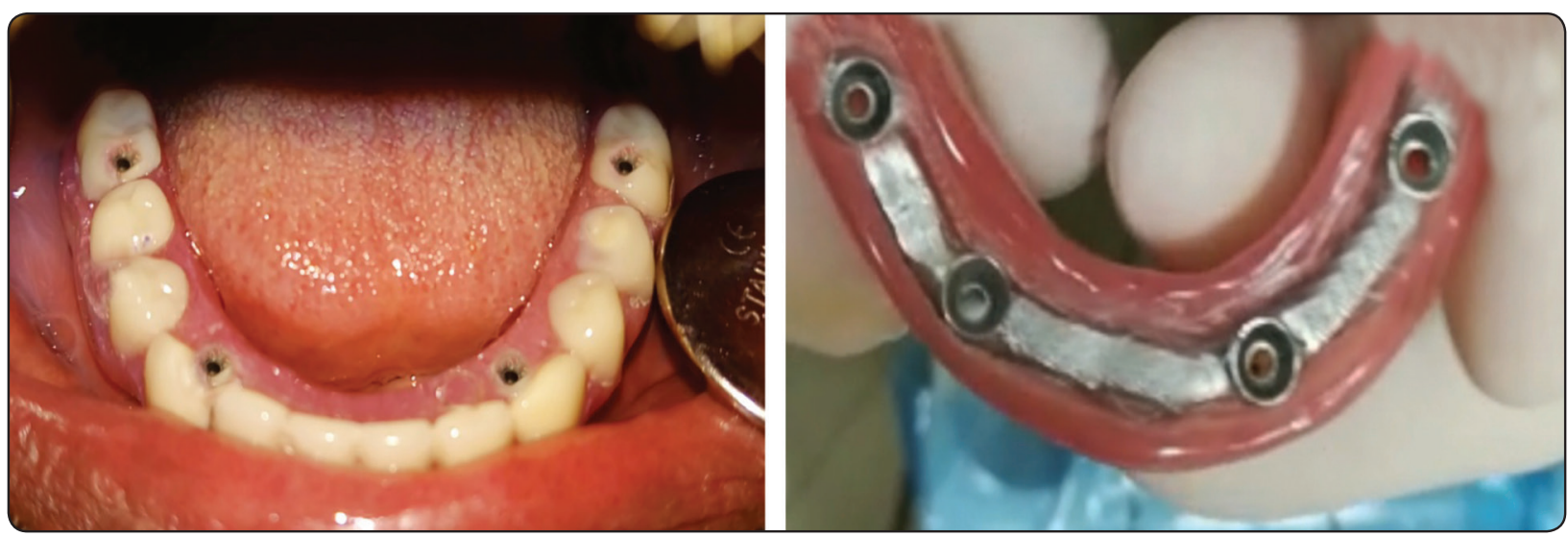

Fig. (5) Group II (Inclined implants, non-cantilevered prosthesis), A) Intra-oral view, B) Fitting surface of the prosthesis 


\section{Evaluation of biologic and prosthetic complications}

The following biological complications (on the implant level) were measured ${ }^{16}$ : Implant loss, implant survival and success, Pain, swelling, abscess, fistulae formation, peri-implantitis, peri-implant mucositis., mucosal bleeding, pocket depths $>5 \mathrm{~mm}$, and vertical bone resorption $>2.5 \mathrm{~mm}$. Prosthetic complications include Prosthesis fracture, Ceramic crown fracture, Acrylic crown fracture, Cylinder fracture, artificial gingiva fracture, Abutment fracture, Abutment screw loosening, Prosthetic screw fracture, and Prosthetic screw loosening ${ }^{16}$. Both biological and prosthetic complications were measured after one year from prosthesis insertion.

\section{Statistical analysis}

SPSS ${ }^{\circledR}$ software version 25 (SPSS Inc., Chicago, IL, USA) was used for data analysis. The descriptive statistics of both biologic and prosthetic complications for both groups were calculated in terms of frequency and percentages using frequency distribution (contingency) tables. To test the difference in proportions of biologic and prosthetic complications between groups, the Chi-square test was used. P-values $<0.05$ were considered to be significant.

\section{RESULTS}

Biological complications (on the implant level) of cantilevered (vertical) and non-cantilevered (inclined) implants are presented in table 1. There were no implant failures occurred in both groups and the survival rate was $100 \%$ in cantilevered and non-cantilevered groups. Pain and swelling were associated with one implant $(6.25 \%)$ in each group and were attributed to mucosal irritation by the metal cylinders of the prosthesis. No abscess or fistula formation occurred on the implant level in both groups. There was no significant difference in implant failure, implant survival, pain, swelling, abscess, and fistulae formation between groups $(\mathrm{p}=1.00)$. Peri-implantitis occurred in one implant $(6.25 \%)$ belonged to the non-cantilevered group. However, no significant difference in periimplantitis between groups was noted $(p=.15)$. Peri-implant mucositis occurred in 2 implants $(12.5 \%)$ belonged to the cantilevered group and 4 implants (25\%) in the non-cantilevered group. However, no significant difference in peri-implant mucositis between groups was noted $(\mathrm{p}=.72)$. The incidence of bleeding on probing was 2 implants (12.5\%) and 4 implants (25\%) for cantilevered and non-cantilevered groups respectively with no significant difference between groups $(\mathrm{p}=.36)$. No implants Peri-implant pockets $>5 \mathrm{~mm}$ was noted for the cantilevered group. The incidence of Periimplant pockets $>5 \mathrm{~mm}$ was 4 implants $(25 \%)$ in the non-cantilevered groups. Non-cantilevered groups showed significantly higher pocket depth than the cantilevered group $(\mathrm{p}=.015)$. Marginal bone loss $>2.5 \mathrm{~mm}$ occurred in 4 implants $(25 \%)$ in the cantilevered group and one implant $(6.25 \%)$ in the non-cantilevered group. The cantilevered group showed significantly higher bone loss than the noncantilevered group ( $\mathrm{p}=.033)$.

Prosthetic complications of cantilevered (vertical) and non-cantilevered (inclined) implants were presented in table 2 . There were no prosthesis fractures occurred in both groups and the survival rate of the prosthesis was $100 \%$ in cantilevered and non-cantilevered groups. Artificial acrylic crown fractures occurred in 2 prostheses (50\%) in the cantilevered group and no crown fractures occurred in the non-cantilevered group. The cantilevered group showed significantly higher crown fracture than the non-cantilevered group $(\mathrm{p}=.047)$. Teeth wear occurred in 2 prostheses $(50 \%)$ in the cantilevered group and one prosthesis $(25 \%)$ in the non-cantilevered group. There was no difference in teeth wear between groups $(\mathrm{p}=.45)$. One gingival acrylic fracture $(25 \%)$ occurred in the cantilevered group and no fractures occurred in the non cantilevered group. 
No significant difference in gingival acrylic fractures between groups was noted $(\mathrm{p}=.28)$. No cylinder fracture, abutment fracture, and prosthetic screw fracture occurred in both groups. Abutment screw loosening occurred in 3 implants (18.75\%) belonged to the cantilevered group and no abutment screw loosening occurred in the non-cantilevered group. The cantilevered group showed a significantly higher abutment screw loosening than the non-cantilevered group $(\mathrm{p}=.049)$. Prosthetic screw loosening occurred in 6 implants $(37.5 \%)$ belonged to the cantilevered group and one $(6.25 \%)$ prosthetic screw loosening occurred in the noncantilevered group. The cantilevered group showed a significantly higher prosthetic screw loosening than the non-cantilevered group $(\mathrm{p}=.033)$.

TABLE (1) Biological complications (on the implant level) of cantilevered (vertical) and non-cantilevered (inclined) implants

\begin{tabular}{|l|c|c|c|c|c|}
\hline & \multicolumn{2}{|c|}{$\begin{array}{c}\text { Cantilevered } \\
\text { (Group I) }\end{array}$} & \multicolumn{2}{c|}{$\begin{array}{c}\text { Non cantilevered } \\
\text { (Group II) }\end{array}$} & P-value \\
\cline { 2 - 5 } & Incidence & Percentage & Incidence & Percentage \\
\hline Implant failure & 0 & $0 \%$ & 0 & $0 \%$ & 1.00 \\
\hline Implant survival & 16 & $100 \%$ & 16 & $100 \%$ & 1.00 \\
\hline Pain, swelling & 1 & $6.25 \%$ & 1 & $6.25 \%$ & 1.00 \\
\hline Abscess, fistulae formation & 0 & $0 \%$ & 0 & $0 \%$ & 1.00 \\
\hline Peri-implantitis & 0 & $0 \%$ & 1 & $6.25 \%$ & .15 \\
\hline Per-implant mucositis & 2 & $12.5 \%$ & 4 & $25 \%$ & .72 \\
\hline Bleeding on probing & 2 & $12.5 \%$ & 4 & $25 \%$ & .36 \\
\hline Peri-implant pockets $>\mathbf{5} \mathbf{~ m m}$ & 0 & $0 \%$ & 4 & $25 \%$ & $.015^{*}$ \\
\hline Marginal bone loss $>\mathbf{2 . 5 m m}$ & 4 & $25 \%$ & 1 & $6.25 \%$ & $.033^{*}$ \\
\hline
\end{tabular}

* p is significant at $5 \%$

TABLE (2): Prosthetic complications of cantilevered (vertical) and non-cantilevered (inclined) implants

\begin{tabular}{|l|c|c|c|c|c|}
\hline & \multicolumn{5}{|c|}{ On patient level } \\
\hline Prosthesis fracture & 0 & $0 \%$ & 0 & $0 \%$ & 1.00 \\
\hline Crown fracture & 2 & $50 \%$ & 0 & $0 \%$ & .047 \\
\hline Teeth wear & 2 & $50 \%$ & 1 & $25 \%$ & .45 \\
\hline Gingiva fracture & 1 & $25 \%$ & 0 & $0 \%$ & .28 \\
\hline & \multicolumn{7}{|c|}{ On implant level } & 0 & $0 \%$ \\
\hline Cylinder fracture & 0 & $0 \%$ & 0 & $0 \%$ & 1.00 \\
\hline Abutment fracture & 0 & $0 \%$ & 0 & $0 \%$ & $.049 *$ \\
\hline Abutment screw loosening & 3 & $18.75 \%$ & $0 \%$ & $0 \%$ & 1.00 \\
\hline Prosthetic screw fracture & 0 & 0 & $0 \%$ & $0.25 \%$ & $.033 *$ \\
\hline Prosthetic screw loosening & 6 & $37.5 \%$ & 1 & 0.00 \\
\hline
\end{tabular}




\section{DISCUSSION}

There were no implant failures occurred in both groups and the survival rate was $100 \%$ in cantilevered and non-cantilevered groups. This could be attributed to several factors. Firstly, all implants were inserted in the inter-foraminal area of the atrophied mandible where the dense basal bone is dominant. The increased bone density provides an excellent survival rate of the implants even cantilever extensions were used ${ }^{22}$. Secondly, the delayed loading protocol was used which avoid implant overloading in the critical healing period. This allows more to bone to implant contact to be established without disturbance ad increase the success rate. Thirdly the muscle strength and bite force of the edentulous patient are predominantly reduced in the elderly patients ${ }^{23}$ included in this study. This will transmit a reduced occlusal force to the prosthesis and may contribute to an increased survival rate.

Peri-implantitis occurred in one inclined implant that belonged to the non-cantilevered group. The implant was associated with mucosal inflammation and bone loss. However, the implant was survived and did not require removal. This may be attributed to increased load on the inclined implant. In line with this explanation, in vitro stress analysis reported that inclined implants may be more prone to occlusal overload than bone surrounding implants ${ }^{24}$. This could over time lead to bone level changes and increase the risk for peri-implant diseases ${ }^{24}$. Although no significant difference in incidence of peri-implant mucositis and bleeding on probing between groups was noted, peri-implant mucositis and bleeding on probing occurs more in the non cantilevered group. This could be attributed to the localized unhygienic condition and the increased plaque accumulation around the posterior (angulated) abutments ${ }^{25}$. The increased plaque may be attributed to the difficult access to perform oral hygiene on the inclined implants and the decreased manual dexterity of the aged participants.
A similar finding was noted by Malo et al. ${ }^{26}$ with 4 inclined implants supporting All on four fixed metal-acrylic fixed complete denture. In contrast, a progressive decrease in plaque and bleeding scores were observed for inclined implants supporting All on four fixed prostheses in atrophied edentulous mandible ${ }^{27}$

Non-cantilevered groups (inclined implants) showed significantly higher pocket depth than the cantilevered group (vertical implant). This may be due to the position of the posterior inclined implants in the alveolar bone. The level of the platform of inclined implants is positioned on the level of the alveolar crest at the mesial side, however, on the distal side, the platform is positioned below the level of the crest about $1-1.5 \mathrm{~mm}$. This creates a localized bone defect with increased pocket depth $^{26}$. The cantilevered group (vertical implant) showed significantly higher bone loss than the non-cantilevered group $(\mathrm{p}=.033)$. In line with this observation, Zyl et al ${ }^{28}$ reported that cantilever extension beyond $15 \mathrm{~mm}$ may cause increased stress in the lingual and buccal sides of the implants, which may cause the interfacial strain to reach overload zone and may cause microfracture of the bone, and/or bone resorption. ${ }^{29}$ Also, Sertqoz and Guvener ${ }^{30}$ reported that stresses at the bone/implant interfaces were maximized at the most distal bone/ implant interface on the loaded sides; and stresses significantly increased with cantilever length. On the other hand, the decreased bone loss on the inclined (non-cantilevered group) may be due to tilted implants are associated with lower absolute values of stresses compared with the non-tilted implants, indicating a possible biomechanical advantage of titling implants in reducing stresses at the boneimplant interface ${ }^{31}$. Moreover, the use of tilted implants increased the anterior-posterior spread, splinting the implants with a rigid superstructure may contribute to a favorable pattern of bone resorption regardless of tilted implant placement 32. In agreement with our findings, another study 
reported that distal tilting of posterior implants in splinted, full, fixed prostheses without cantilevers can reduced stress generated around peri-implant bone when compared to stress levels seen in periimplant bone with vertical implants and cantilevered segments in similar full fixed prostheses ${ }^{33}$. Similarly, Horita, Sugiura et al. ${ }^{34}$ reported that mandibular fixed full-arch prostheses without cantilevers may result in a favorable reduction of the peri-implant bone strain during the healing period, compared with cantilevers. They added that the high compressive stress in the 15 -mm cantilever models causing a $33 \%$ increase in stresses compared with the 5-mm cantilevers. In contrast to our finding, Krennmair et al. ${ }^{35}$ noted no significant difference in marginal bone loss between patients with four ( 2 anterior/2 posterior) axial implants and patients with 2 anterior axial/2 distal tilted implants. The difference in the results may be due to both axial and tilted implants groups in the study of Krennmair et al. included distal cantilevers (Axial $=15.7 \mathrm{~mm}$ and tilted $=13.7 \mathrm{~mm}$ ). However, in this study, no cantilevers were used in the tilted implant group.

There were no prosthesis fractures occurred in both groups and the survival rate of the prosthesis was $100 \%$. In contrast, Zarb and Jansson noted that implant frameworks were vulnerable to fracture, especially at the junctions between distal abutments and cantilevered segments ${ }^{36}$. The reduced prosthetic fracture in our study may be due to the use of acrylic resin which has a cushion effect to the applied load. Moreover, the increased thickness of the metal bar reinforces the acrylic prosthesis and prevent fracture of the prosthesis compared to the metal-ceramic prosthesis that tends to fracture at the junction of distal abutments and cantilevers. The cantilevered group showed significantly higher crown fracture than the non-cantilevered group. Moreover, teeth wear and gingival acrylic fracture occurred more in the cantilevered group than the non-cantilevered group although the difference was not significant. Similarly, tooth fracture was reported as the main prothetic complication after a 10-year prospective study for implant-supported maxillary, full-arch acrylic prostheses ${ }^{37}$. The increased acrylic teeth wear and fracture may be due to axial implants are located more anteriorly in the cantilevered group. This may encourage the patients to incise anteriorly with maximum bite force $^{38}$ creating a similar biomechanical situation to the distal extension removable partial denture ${ }^{39}$. The increased bite forces on the anterior teeth of the prosthesis may induce acrylic teeth wear and fracture that usually occurred on the anterior teeth. Another explanation is the reduced prosthetic space for acrylic resin caused by the increased thickness of the metal bar which may facilitate acrylic teeth separation and fracture. However, the problem was easy to be solved by addition of new teeth of the same shade using autopolymerized acrylic resin.

No cylinder fracture, abutment fracture, and prosthetic screw fracture occurred in both groups. In line with our finding, no abutment or abutment screw fractures were reported for implant-supported maxillary, full-arch acrylic prostheses ${ }^{37}$. Conversely, Zarb and Schmitt reported clinical problems that included: abutment screw fracture, gold alloy retaining screw fracture, that occurred especially with cantilevered portions of the hybrid prosthesis. ${ }^{40}$ However, these complications are usually associated with the presence of natural teeth in the opposing jaw or with increase occlusal force factor caused by increased strength of the masticatory muscles (young patients) or the inclusion of patients with bruxism. Another explanation may be attributed to the opposing dentition which is conventional dentures in both groups thus transmitting reduced forces to the implant compared to natural dentition or fixed prosthesis during function and parafunction ${ }^{41}$. Also, the acrylic teeth had a cushion (shock-absorbing) effect. All these factors resulted in a significant reduction of occlusal forces and the absence of cylinder, abutment, and prosthetic screw fractures. 
The cantilevered group showed a significantly higher abutment screw loosening and prosthetic screw loosening than the non-cantilevered group. This could be attributed to the unfavorable occlusal loading on the cantilever extension that was reported to cause loosening of abutment and prosthetic screws and prosthetic posts, framework fracture, and implant loss. In this study, the mean length of the cantilever $=14.2 \mathrm{~mm}$ which is relatively high as it has been suggested that the extension from the midpoint of the most distal implant must not exceed $15 \mathrm{~mm}$ in the mandible ${ }^{7}$. In line with our findings, McAlarney and Stavropoulos ${ }^{42}$ reported that loading cantilevers increased loads distributed to implants in full-arch clinical cases which could lead to increased prosthetic screw loosening. Similarly, Baldin Able et al. ${ }^{43}$ reported screw loosening among the common prosthetic complications of cantilevered hybrid mandibular fixed completearch dental prostheses. However, they added that cantilever length was not associated with significant prosthetic complications. The limitation of this study included the small sample size and the short follow up period.

\section{CONCLUSION}

Within the limits of this short term clinical trial, both cantilevered and non-cantilevered fixed hybrid metal acrylic prostheses can be used successfully for vertical and inclined implants respectively in atrophied mandible with favorable biologic and prosthetic outcomes after one year. However, with exception of increased pocket depth, the noncantilevered group was advantageous regarding reduced bone loss, crown fracture, abutment, and prosthetic screw loosening, compared to the cantilevered group.

\section{REFERENCES}

1. Attard NJ, Zarb GA. Long-term treatment outcomes in edentulous patients with implant-fixed prostheses: the Toronto study. Int J Prosthodont. 2004;17:417-24.
2. Santagata M, Guariniello L, D'Andrea A, Tartaro G. A modified crestal ridge expansion technique for immediate placement of implants: a report of three cases. J Oral Implantol. 2008;34:319-24.

3. Stellingsma C, Vissink A, Meijer HJ, Kuiper C, Raghoebar GM. Implantology and the severely resorbed edentulous mandible. Crit Rev Oral Biol Med. 2004;15:240-8.

4. Stellingsma K, Raghoebar GM, Meijer HJ, Stegenga B. The extremely resorbed mandible: a comparative prospective study of 2-year results with 3 treatment strategies. Int J Oral Maxillofac Implants. 2004;19:563-77.

5. Branemark. I, Zarb. G, Albrektsson. T. Tissue Integrated Prostheses. Chicago, Quintessence. 1985:pp 51-70, 117-I28.

6. Adell R, Lekholm U, Rockler B, Branemark PI. A 15-year study of osseointegrated implants in the treatment of the edentulous jaw. Int J Oral Surg. 1981;10:387-416.

7. Jain AR, Nallaswamy D, Ariga P, Philip JM. Full mouth rehabilitation of a patient with mandibular implant screw retained Fp-3 prosthesis opposing maxillary acrylic removable over-denture. Contemp Clin Dent. 2013;4: 231-5.

8. McAlarney ME, Stavropoulos DN. Theoretical cantilever lengths versus clinical variables in fifty-five clinical cases. The Journal of prosthetic dentistry. 2000;83:332-43.

9. Drago C. Ratios of Cantilever Lengths and Anterior-Posterior Spreads of Definitive Hybrid Full-Arch, Screw-Retained Prostheses: Results of a Clinical Study. J Prosthodont. 2018;27:402-8

10. Malo P, de Araujo Nobre M, Lopes A, Francischone C, Rigolizzo M. "All-on-4” immediate-function concept for completely edentulous maxillae: a clinical report on the medium (3 years) and long-term (5 years) outcomes. Clin Implant Dent Relat Res. 2012;14 Suppl 1:e139-50.

11. Malo P, Friberg B, Polizzi G, Gualini F, Vighagen T, Rangert B. Immediate and early function of Branemark System implants placed in the esthetic zone: a 1-year prospective clinical multicenter study. Clin Implant Dent Relat Res. 2003;5 Suppl 1:37-46.

12. Malo P, Rangert B, Nobre M. All-on-4 immediate-function concept with Branemark System implants for completely edentulous maxillae: a 1-year retrospective clinical study. Clin Implant Dent Relat Res. 2005;7 Suppl 1:S88-94.

13. Malo P, Rangert B, Nobre M. "All-on-Four" immediatefunction concept with Branemark System implants for completely edentulous mandibles: a retrospective clinical study. Clin Implant Dent Relat Res. 2003;5 Suppl 1:2-9. 
14. Pozzi A, Sannino G, Barlattani A. Minimally invasive treatment of the atrophic posterior maxilla: a proof-ofconcept prospective study with a follow-up of between 36 and 54 months. J Prosthet Dent. 2012;108:286-97.

15. Francetti L, Agliardi E, Testori T, Romeo D, Taschieri S, Del Fabbro M. Immediate rehabilitation of the mandible with fixed full prosthesis supported by axial and tilted implants: interim results of a single cohort prospective study. Clin Implant Dent Relat Res. 2008;10:255-63.

16. Maló P, de Araújo Nobre M, Lopes A, Ferro A, Botto J. The All-on-4 treatment concept for the rehabilitation of the completely edentulous mandible: A longitudinal study with 10 to 18 years of follow-up. Clin Implant Dent Relat Res. 2019.

17. Link-Bindo EE, Soltys J, Donatelli D, Cavanaugh R. Common Prosthetic Implant Complications in Fixed Restorations. Compend Contin Educ Dent. 2016;37:431-6;quiz9.

18. Cawood JI, Howell RA. A classification of the edentulous jaws. Int J Oral Maxillofac Surg. 1988;17:232-6.

19. Jensen OT, Adams MW, Cottam JR, Parel SM, Phillips WR. The all on 4 shelf: mandible. J Oral Maxillofac Surg. 2011;69:175-81.

20. Mericske-Stern RD, Taylor TD, Belser U. Management of the edentulous patient. Clin Oral Implants Res. 2000;11 Suppl 1:108-25.

21. Sannino G, Bollero P, Barlattani A, Gherlone E. A Retrospective 2-Year Clinical Study of Immediate Prosthetic Rehabilitation of Edentulous Jaws with Four Implants and Prefabricated Bars. J Prosthodont. 2017;26:387-94.

22. Rangert BO, Jemt T. Forces and Moments on Brånemark Implants. International Journal of Oral \& Maxillofacial Implants. 1989;4.

23. ELsyad MA, Ibrahim AE, Nawar NHH, Belal TM. Electromyographic Connectivity of Masseter Muscle with Different Retentive Attachments for Implant Overdentures in Patients with Atrophied Mandibular Ridges: A Crossover Study. Int J Oral Maxillofac Implants. 2019;34:1213-22.

24. Begg T, Geerts GA, Gryzagoridis J. Stress patterns around distal angled implants in the all-on-four concept configuration. Int J Oral Maxillofac Implants. 2009;24:663-71.

25. ELsyad MA, Alameldeen HE, Elsaih EA. Four-implantsupported fixed prosthesis and milled bar overdentures for rehabilitation of the edentulous mandible: A 1-year randomized controlled clinical and radiographic study. Int J Oral Maxillofac Implants. 2019;34:1493-503.
26. Malo P, de Araujo Nobre M, Lopes A. The use of computer-guided flapless implant surgery and four implants placed in immediate function to support a fixed denture: preliminary results after a mean follow-up period of thirteen months. J Prosthet Dent. 2007;97:S26-34.

27. Weinstein R, Agliardi E, Fabbro MD, Romeo D, Francetti L. Immediate rehabilitation of the extremely atrophic mandible with fixed full-prosthesis supported by four implants. Clin Implant Dent Relat Res. 2012;14:434-41.

28. van Zyl PP, Grundling NL, Jooste $\mathrm{CH}$, Terblanche E. Three-dimensional finite element model of a human mandible incorporating six osseointegrated implants for stress analysis of mandibular cantilever prostheses. Int J Oral Maxillofac Implants. 1995;10:51-7.

29. Malhotra AO, Padmanabhan TV. Load transfer in tilted implants with varying cantilever lengths in an all-on-four situation. Australian dental, 2012. 2012.

30. Sertgoz A, Guvener S. Finite element analysis of the effect of cantilever and implant length on stress distribution in an implant-supported fixed prosthesis. J Prosthet Dent. 1996;76:165-9.

31. Bellini CM, Romeo D, Galbusera F. A finite element analysis of tilted versus nontilted implant configurations in the edentulous maxilla.. International, 2009. 2009.

32. Krennmair S, Weinländer M, Malek M, Forstner T, Krennmair G, Stimmelmayr M. Mandibular Full-Arch Fixed Prostheses Supported on 4 Implants with Either Axial Or Tilted Distal Implants: A 3-Year Prospective Study. Clin Implant Dent Relat Res. 2016;18:1119-33.

33. Bevilacqua M, Tealdo T, Pera F, Menini M, Mossolov A, Drago $\mathrm{C}$ et al. Three-dimensional finite element analysis of load transmission using different implant inclinations and cantilever lengths. Int J Prosthodont. 2008;21:539-42.

34. Horita S, Sugiura T, Yamamoto K, Murakami K, Imai Y, Kirita T. Biomechanical analysis of immediately loaded implants according to the "All-on-Four" concept. Journal of prosthodontic research. 2017;61:123-32.

35. Krennmair S, Weinlander M, Malek M, Forstner T, Krennmair G, Stimmelmayr M. Mandibular Full-Arch Fixed Prostheses Supported on 4 Implants with Either Axial Or Tilted Distal Implants: A 3-Year Prospective Study. Clin Implant Dent Relat Res. 2016;18:1119-33.

36. Zarb GA, Albrektsson T. Tissue-integrated prostheses: osseointegration in clinical dentistry: Quintessence Pub Co; 1985. 
37. Fischer K, Stenberg T. Prospective 10-year cohort study based on a randomized, controlled trial (RCT) on implantsupported full-arch maxillary prostheses. part II: prosthetic outcomes and maintenance. Clin Implant Dent Relat Res. 2013;15:498-508.

38. Thiel CP, Evans DB, Burnett RR. Combination syndrome associated with a mandibular implant-supported overdenture: a clinical report. J Prosthet Dent. 1996;75:107-13.

39. 39. Barber HD, Scott RF, Maxson BB, Fonseca RJ. Evaluation of anterior maxillary alveolar ridge resorption when opposed by the transmandibular implant. J Oral Maxillofac Surg. 1990;48:1283-7.

40. Hemmings KW, Schmitt A, Zarb GA. Complications and maintenance requirements for fixed prostheses and over- dentures in the edentulous mandible: a 5-year report. International Journal of Oral \& Maxillofacial Implants. 1994;9.

41. Tashkandi EA, Lang BR, Edge MJ. Analysis of strain at selected bone sites of a cantilevered implant-supported prosthesis. J Prosthet Dent. 1996;76:158-64.

42. McAlarney ME, Stavropoulos DN. Theoretical cantilever lengths versus clinical variables in fifty-five clinical cases. J Prosthet Dent. 2000;83:332-43.

43. Able FB, de Mattias Sartori IA, Thome G, Moreira Melo AC. Retrospective, cross-sectional study on immediately loaded implant-supported mandibular fixed completearch prostheses fabricated with the passive fit cementation technique. J Prosthet Dent. 2018;119:60-6. 